\title{
Improving antibiotic prescribing for surgical prophylaxis - the role of perioperative nurses
}

Follow this and additional works at: https://www.journal.acorn.org.au/jpn

Part of the Health Services Administration Commons, Health Services Research Commons, Perioperative, Operating Room and Surgical Nursing Commons, and the Surgery Commons

(c) (†)

This work is licensed under a Creative Commons Attribution 4.0 License.

\section{Recommended Citation}

Nicholson, Pat (2019) "Improving antibiotic prescribing for surgical prophylaxis - the role of perioperative nurses," Journal of Perioperative Nursing: Vol. 32 : Iss. 1 , Article 2.

Available at: https://doi.org/10.26550/2209-1092.1056

https://www.journal.acorn.org.au/jpn/vol32/iss1/2

This Article is brought to you for free and open access by Journal of Perioperative Nursing. It has been accepted for inclusion in Journal of Perioperative Nursing by an authorized editor of Journal of Perioperative Nursing. 


\section{Improving antibiotic prescribing for surgical prophylaxis - the role of perioperative nurses}

Surgical site infection is a potential post-surgery risk that needs to be managed effectively as part of good patient care. The discovery of antibiotics in the $20^{\text {th }}$ century and their associated use as surgical antibiotic prophylaxis, often with other interventions such as oxygenation, glycaemic control and surgical antisepsis, has minimised this procedural burden.

However, the global increase in antimicrobial resistance (AMR) is limiting the effectiveness of antibiotics currently available when treating infections and impacting on the delivery of safe and effective care for patients. As a result, many infections are no longer responsive to first line antibiotic choices. The overuse and misuse of antibiotics, wherever this occurs, impacts the efficacy of surgical antibiotic prophylaxis. This, compounded by the decreased antibiotic development pipeline, means that managing an infection is no longer as simple as just selecting 'another antibiotic'.

Due to AMR, complex infections are now being treated with potentially more toxic, costly and complicated regimens than in the past. This creates additional risks for patients, including potentially adverse outcomes from the antibiotics used and increased length of hospital stay due to a lack of oral therapeutic choices. Patients with unnecessary exposure to long courses of antibiotic prophylaxis are also at a higher risk of morbidity and mortality if they develop an infection as it is more likely the organism will be resistant to commonly prescribed antibiotics.
The Australian Commission on Safety and Quality in Health Care (the Commission) coordinates the Antimicrobial Use and Resistance in Australia (AURA) Surveillance System, which provides a range of AMR and antibiotic use surveillance data. AURA also provides a platform for voluntary standardised audits of surgical prophylaxis through the Hospital National Antimicrobial Prescribing Survey (NAPS).

Data from participating hospitals in 2017 showed that 30.5 per cent of surgical prophylaxis prescriptions for inpatients extended 24 hours beyond the time of surgery. This is despite guidelines recommending surgical prophylaxis durations of less than 24 hours. Commonly, surgical antibiotic prophylaxis was found to be too broad or too narrow for the organisms known to cause surgical site infections or to be inconsistent with guidelines (with no indication of patient characteristics that would require variation), or the wrong dose was prescribed.

Variation in surgical antibiotic prophylaxis prescription often occurs because of individual prophylaxis preferences. Despite evidence to the contrary ${ }^{1,2}$, the perception that adverse outcomes are reduced with longer and broader spectrum antibiotic intravenous courses still exists. Topical or deep surgical site administration has also been reported.

The increased health care-associated complications of prolonged or novel intra-operative antibiotic use (for example irrigations, pastes or washes) also need to be considered,

\section{Authors}

Robert Herkes

MBBS FRACP FCICM

Chief Medical Officer, Australian

Commission on Safety and Quality in Health Care, Sydney NSW Australia

Associate Professor Pat Nicholson PhD, RN, FACORN

School of Nursing and Midwifery, Centre for Quality and Patient Safety Research, Faculty of Health, Deakin University, Geelong Vic Australia

particularly where the evidence base for alternative practices is limited.

Process issues still account for many variations from guidelines-based practice. Improved standardisation could bring practice more in line with consistent and reliable delivery of antibiotic prophylaxis. There are many opportunities for improvement including:

- consistency in documentation of fixed antibiotic duration

- development of and adherence to evidence or consensus-based guidelines

- optimising administration timing for optimal concentration of antibiotics during the surgical procedure.

The timing of prophylactic antibiotics is crucial, and nurses working in the perioperative setting are well placed to have a significant impact on this aspect of surgical antibiotic prophylaxis. Optimal timing is dependent on the pharmacokinetics of the antibiotic used to optimise tissue concentrations. Vancomycin (and antibiotics with a longer half-life) should be commenced within 120 minutes of knife to skin; the infusion does not have to be completed prior to the commencement of surgery. vancomycin can cause red man syndrome when administered too quickly in an attempt to finish the infusion prior to knife to skin. 
The timing of antibiotic administration also requires logistic coordination of the patient's journey from the ward to the operating suite and from the Post Anaesthesia Care Unit back to the ward. Nurses can also support best practice by promoting documentation of the plan for surgical antibiotic prophylaxis to avoid confusion when the patient returns to the ward. Prolonged administration of intravenous surgical prophylaxis can also increase the risk of a cannula site infection.

Simple changes such as promoting the importance of correct surgical antimicrobial prophylaxis for every procedure could also increase consistent administration and improve choice practices. Clarity regarding the lead in the choice of antibiotic (anaesthetic and surgical specialties) may also aid in more consistent administration practices ${ }^{3}$.

Under the National Safety and

Quality Health Service (NSQHS)

Standards, every hospital is required to have a local antimicrobial stewardship program to optimise use of antimicrobials and improve the use of surgical antimicrobial prophylaxis within hospitals. Nurses are extremely valuable in their participation in multidisciplinary efforts to facilitate audits and feedback procedures or drive dedicated quality improvement projects. The provision of safe and effective care to patients is the ultimate goal. To achieve this, the risks and benefits of antimicrobial use need to be balanced.

The Commission is working with ACORN to provide perioperative nurses with resources to assist in safe antimicrobial use. Go to www.safetyandquality.gov.au/SAP to find out how you can improve surgical antibiotic prophylaxis in your organisation.

\section{References}

1. Harbarth S, Samore MH, Lichtenberg D, Carmeli Y. Prolonged antibiotic prophylaxis after cardiovascular surgery and its effect on surgical site infections and antimicrobial resistance. Circulation 2000;101(25):29162921

2. Broom J, Broom A, Kirby E, Post JJ. Improvisation versus guideline concordance in surgical antibiotic prophylaxis: A qualitative study. Infection 2018;46(4): 541-548.

3. Charani E, Tarrant C, Moorthy K, Sevdalis $\mathrm{N}$, Brennan L, Homes AH. Understanding antibiotic decision making in surgery - a qualitative analysis. Clin Microbiol Infect 2017;23(10):752-760. 